\title{
Externalismo y autoridad de la primera persona: McKinsey vs. Davidson ${ }^{1}$
}

\author{
Marc Jiménez Rolland \\ Departamento de Filosfía \\ Universidad Autónoma de Aguascalientes \\ marcji2121@yahoo.com.mx
}

\section{Introducción}

Desde hace poco más de tres décadas se introdujo en la discusión filosófica de la tradición analítica una tentativa sumamente ambiciosa: la del desarrollo de una tesis externalista acerca del contenido. Podemos enunciar lo que establece dicha tesis de la siguiente manera:

(E): El contenido de los enunciados mentales intencionales de un individuo depende, al menos en parte, de relaciones entre ese individuo y su entorno; en otras palabras, la individuación de pensamientos amplios ${ }^{2}$ depende necesariamente de las relaciones que una persona guarda con su entorno físico y, en algunos casos, social [esto es, su contexto histórico o las prácticas de la comunidad lingüística a la que pertenece] (ver Ludlow \& Martin 1998, 1; Burge 1988, 111; Gertler 2003).

\footnotetext{
${ }^{1}$ Versiones preliminares de este ensayo fueron presentadas en el Coloquio de Estudiantes de Maestría de la Universidad Autónoma de Zacatecas, en junio de 2008, y en el Seminario de Profesores de Filosofía de la Universidad Autónoma de Aguascalientes, el 13 febrero de 2009. Agradezco especialmente al Dr. Jorge R. Tagle Marroquín, por su invaluable apoyo, y al Mtro. Francisco Serrano, por sus útiles comentarios; asimismo, estoy en deuda con los profesores y alumnos de la UAA por sus perspicaces observaciones al texto.

${ }^{2}$ Retomo esta forma de presentación de las actitudes proposicionales (creencias, deseos, etc.) que expresan contenido amplio (esto es, refieren a propiedades extrínsecas) del texto de Putnam 1975, quien las distingue de pensamientos en sentido estrecho (dolor, cosquilleo, entre otros), que presumiblemente carecen de contenido amplio (en tanto refieren a propiedades intrínsecas). En la discusión subsiguiente no me ocuparé de este último tipo de pensamientos.
} 
Aunque los argumentos en favor de esta postura -incluso en su formulación más débil- no son aún concluyentes, se exploran ya las consecuencias que tendría en otros campos si fuera verdadera. Uno de los ámbitos en los que parece tener especial relevancia es el relativo al conocimiento de los propios estados mentales (AC), pues existe cierta tensión entre esta idea y (E). Ludlow \& Martin indican esta tensión a través del cuestionamiento sobre las implicaciones que tendría (E) respecto a la doctrina de que podemos tener un conocimiento a priori acerca de nuestros estados mentales (1998; 1 ). De entrada, parece que (E) se ubica en las antípodas de la concepción cartesiana de esta forma de conocimiento. Sin embargo, la de Descartes no es la única - ni, hasta la fecha, la más plausible - presentación del autoconocimiento (self-knowledge).

Gertler (2003) precisa que hay al menos tres distintas perspectivas al momento de definir en qué consiste el privilegio de la primera persona en el autoconocimiento:

(AC1): El conocimiento de los propios estados mentales es especialmente seguro epistémicamente.

(AC2): Se emplea un método único y especial para determinar el contenido de los propios estados mentales.

(AC3): Las autoadscripciones de estados mentales conllevan una autoridad o suposición de verdad de un tipo especial.

Este mismo autor señala que casi todos aquellos que aceptan el externalismo acerca del contenido afirman que la tensión entre (E) y (AC) es sólo aparente; pocos están dispuestos a abandonar por completo la idea de un conocimiento privilegiado de primera persona (ver Gertler 2003). Algunos otros han considerado que (E) y (AC) son mutuamente excluyentes $y$, por ende, si ha de aceptarse una de estas tesis, deberá rechazarse la otra. ${ }^{3}$ Entre estos autores encon-

\footnotetext{
${ }^{3}$ Existen otras alternativas para evitar la incompatibilidad: rechazar ambas tesis o introducir una tercera ad hoc que elimine la tensión. Agradezco la oportuna precisión sobre este punto al doctor Jorge R. Tagle Marroquín. Puesto que se trabaja bajo el supuesto de que (E) es verdadera, no se considera la primera de estas alternativas; Parent 2007 parece optar por la segunda. El argumento de McKinsey que se expone en la siguiente sección no toma en cuenta esta última posibilidad. Esto podría debilitar la conclusión que establezco en este escrito, pero el
} 
tramos a Michael McKinsey, quien ocupará especialmente nuestra atención en lo que sigue.

\section{El debate sobre la [in]compatibilidad entre (E) y (AC)}

El debate en torno al externalismo sobre el contenido inició a raíz de algunas observaciones semánticas que apoyan las teorías de la referencia directa de nombres, pronombres, deícticos y términos de clase natural. Putnam (1975) señaló que el contenido de los pensamientos de un individuo depende al menos en parte de elementos del entorno físico 'externos' a ese individuo (propiedades físicas extrínsecas al individuo); obtuvo esta conclusión a partir del afamado caso problema de la 'Tierra Gemela'.

Burge (1979), a partir de experimentos mentales similares - que tienen, frente al de Putnam, la ventaja adicional de hacer un uso relativamente inocuo de consideraciones contrafácticas, evitando problemas que oscurecían la formulación original ${ }^{4}-$, añadió a esto que la comunidad de hablantes de la que forma parte el individuo es también relevante en la determinación del contenido de sus pensamientos. Esos experimentos, como explica Burge (1988; 112), mantienen constante la historia causal de una persona (su movimiento corporal, sus estímulos superficiales y su química interna; pero también los enunciados con los que describiría sus estados mentales amplios), esto es, sus propiedades intrínsecas, variando el entorno social en el que la persona interactúa (propiedades extrínsecas). A

tema requiere una mayor consideración.

${ }^{4}$ Me refiero específicamente a elementos desconcertantes en el planteamiento de Putnam 1975, quien nos pide que imaginemos un mundo físicamente idéntico al actual (la Tierra Gemela) con la diferencia de que en él se encuentra el compuesto XYZ con las mismas propiedades superficiales del agua en sustitución de lo que en el mundo actual es H2O. Asimismo, nos pide que consideremos la existencia en ese mundo de un sujeto $S$ físicamente idéntico a un habitante del mundo actual. Esto suscita una inquietud adicional: ¿cómo puede ese sujeto ser físicamente idéntico a un habitante del mundo actual, si cerca de $60 \%$ de su constitución física debe suplantarse por XYZ en lugar de $\mathrm{H} 2 \mathrm{O}$ ? Parecería que tenemos que aceptar parte importante de lo que el funcionalismo quiere mostrar para que el ejemplo tenga sentido. Creo que el problema no afecta de manera importante el planteamiento de Putnam, pero sin duda oscurece sus implicaciones y el alcance de éstas. Tengo noticia de que John McDowell y Philip Pettit han hecho consideraciones al respecto similares a las que apunto aquí, aunque desconozco los detalles de su exposición. 
partir de esto luego se muestra que algunos de los pensamientos de la persona varían. Esta variación indica que, en el mejor de los casos, deben considerarse principios de individuación de tipos mentales a partir de propiedades sociales extrínsecas a la persona en cuestión. Burge consigna esta conclusión parafraseando un célebre pasaje de John Donne: «No man's intentional mental phenomena are insular. Every man is a piece of the social continent, a part of the social main» $(1979 ; 41)$.

La moraleja de los casos propuestos por Putnam (1975) y Burge (1979) es que tenemos muy buenas razones para creer que (E) es verdadera; algunos afirmarían más: la moraleja es que el significado es una propiedad relacional, ergo para conocer el contenido de nuestros estados mentales amplios debemos conocer propiedades extrínsecas. ${ }^{5}$ Aunado a esto, Burge después (1988) afirma que (E) es compatible con una versión de (AC) que involucra «acceso privilegiado» a los propios estados mentales. Es en este punto en donde interviene McKinsey, con su famosa reductio ad absurdum, para abogar en pro de la incompatibilidad (1995; 2002a; 2002b;6 2007).

McKinsey comienza por ofrecer una versión de (AC):

$\left(\mathrm{AC}^{*}\right)$ : Es necesariamente verdadero que si una persona $x$ está pensando que $p$, entonces $x$ puede, en principio, saber a priori que él mismo [o ella misma] está pensando que $p$.

Puede decirse sin mucho problema que $\left(\mathrm{AC}^{*}\right)$ es equivalente a (AC2), en la medida en que invoca un método especial para conocer el contenido de los propios estados mentales, distinto al empleado en la observación sensorial o en la investigación empírica. No obstante, $\left(\mathrm{AC}^{*}\right)$ no es necesariamente similar a (AC1), pues - en tanto McKinsey sigue a Kripke en la distinción entre 'a priori' y 'necesario'

\footnotetext{
${ }^{5}$ Hay una debilidad en esta segunda formulación: una propiedad relacional podría no ser necesariamente lo opuesto a una propiedad intrínseca; muchas propiedades parecen ser tanto relacionales como intrínsecas (ver Wheatherson 2006), por lo que este argumento sería non sequitur. Sin embargo, este tema sigue sujeto a discusión.

${ }^{6}$ La reconstrucción en términos de propiedades relacionales presentada en esta reseña parece vulnerable a las observaciones señaladas en la nota anterior. Sin embargo, no deseo hacer de esto una objeción a McKinsey, pues no se trata de una presentación crucial de la reductio.
} 
(1972; 38-42) - $\left(\mathrm{AC}^{*}\right)$ no especifica ningún tipo especial de seguridad o garantía epistémica. A esto McKinsey suma una formulación de (E) claramente semántica:

(ES): Muchos predicados estructurados de dicto de la forma 'está pensando que $p$ ' expresan propiedades que son amplias, en el sentido de que la posesión de tal propiedad por parte de un agente 'implica lógicamente' la existencia de objetos contingentes o sustancias de cierto tipo que son externas al agente (McKinsey 2002a, 35; 2007).

De modo que, si ponemos a trabajar estas tesis como McKinsey sugiere, tenemos que para «Óscar está pensando que el oro es un metal precioso», de acuerdo con $\left(\mathrm{AC}^{*}\right)$ podemos decir:

(I) Óscar puede saber a priori que está pensando que el oro es un metal precioso.

Pero, dado que «está pensando que el oro es un metal precioso» expresa una propiedad lógicamente amplia, se sigue de (ES) que:

(II) La proposición que Óscar está pensando ['el oro es un metal precioso'] lógicamente implica la proposición $E$,

donde $E$ es una «proposición externa» que asevera o implica la existencia de objetos externos a Óscar (e. g., la proposición de que el oro existe). De modo que la conjunción de (I) y (II) es claramente absur$\mathrm{da}$, pues - a menos que se le atribuyan poderes extraordinarios a la mente y se extienda muy ampliamente el campo de lo que puede conocerse a priori- no es posible que Óscar pueda saber independientemente de cualquier experiencia que el oro existe. Afirmar lo contrario sería una suerte de reivindicación del «argumento ontológico» al estilo de San Anselmo, con todo el amargo sabor que esta reminiscencia conlleva (McKinsey 2002a; 35). Luego, $-\left[(\mathrm{E}) \wedge\left(\mathrm{AC}_{2}\right)\right]$.

$\mathrm{Si}$ hemos de elegir entre rechazar $(\mathrm{E})$ o $\left(\mathrm{AC}_{2}\right)$, sigue McKinsey, no hay duda: rechacemos ( $\left.\mathrm{AC}_{2}\right)$, puesto que tenemos mejores argumentos para (E). De modo que para conocer el contenido de nuestros pensamientos deberíamos investigar empíricamente nuestro 
entorno físico y social. Y esto también da cuenta de $(\mathrm{AC} 1)$, pues la investigación empírica no satisface los requerimientos que esta tesis postula.

Una alternativa que han tomado los defensores de Burge para evitar estas conclusiones es matizar (E) de modo que no tenga como consecuencia (ES) y, por ende, de modo que la incompatibilidad con (AC) no sea la conclusión necesaria. Esta parece ser la línea seguida por McLaughlin \& Tye (1998). Sin embargo, McKinsey ha señalado enfáticamente que esta estrategia no es adecuada, pues ignora que lo que los ejemplos de Putnam y Burge plausiblemente muestran es (ES) $(2002 \mathrm{a}, 35 ; 2007){ }^{7}$

De modo quela reductio de McKinsey prueba que $\longleftarrow\left\{(\mathrm{E}) \wedge\left[\left(\mathrm{AC}_{2}\right) \vee\right.\right.$ $\left.\left.\left(\mathrm{AC}_{1}\right)\right]\right\}$ (ver n. 2). Si aceptamos los argumentos a favor de (E) hemos de rechazar tanto $\left(\mathrm{AC}_{2}\right)$ como $\left(\mathrm{AC}_{1}\right)$ y no sólo una de ellas. Pero, ¿qué hay de $\left(\mathrm{AC}_{3}\right)$ ? ¿Cómo afecta la reductio de McKinsey a la concepción davidsoniana de $(\mathrm{AC})$ ?

En la única ocasión en que discute la postura de Davidson, McKinsey no logra establecer una respuesta contundente en su contra, se limita a considerar que la versión de (AC) defendida por Davidson es muy débil y a sugerir que no trata la discusión en los términos de Putnam. Claramente Davidson no quiere hacer esto. ${ }^{8}$ McKinsey parece, más bien, no haber comprendido en absoluto el punto de Davidson (1995; 176-8).

Davidson se centra en el aspecto lingüístico del problema de la autoridad de la primera persona, aunque permanece en el paradigma tradicional retomando la suposición cognitiva (i.e., una persona normalmente sabe lo que cree), asume que «si la autoridad de la primera persona en el lenguaje puede ser explicada, habremos hecho mucho, si no todo, de lo que debe ser hecho para caracterizar y dar cuenta de los hechos epistemológicos» $(1984 ; 102)$. Para clarificar la

\footnotetext{
${ }^{7}$ En este sentido McKinsey señala: «the compatibilist metaphysical strategy effectively removes the semantic basis of externalism, and so takes away whatever reason there was to believe externalism in the first place» (2002a, 35; cursivas mías). Por ello, de aquí en delante emplearé indistintamente (E) para referir a (ES).

${ }^{8}$ Esto es especialmente evidente en Davidson (1987; 113-134), respecto al compromiso ontológico con propiedades, y en Davidson (1987; 139-140), en lo relativo al aparato semántico de la referencia directa.
} 
autoridad en las proferencias lingüísticas, Davidson recurre a los requisitos de la interpretación. Arguye que uno sabe, al proferir un reporte sobre los propios estados mentales en forma veraz, lo que éste significa, sin importar la fuente o naturaleza de este conocimiento, de modo que, al sostener dicho reporte como verdadero, uno sabe lo que piensa, siente, cree, etc., es posible que el otro no lo sepa, si no entiende lo que se le dice y en esto radica la autoridad. La condición para que el conocimiento sea asequible a un interlocutor es que el hablante sea interpretable, de modo que lo que significan sus palabras sea lo que él pretende que signifiquen.

El tratamiento de Davidson del problema de la autoridad de la primera persona tiene la forma de una inferencia a la mejor explicación. ${ }^{9}$ Su punto de partida es el hecho de que sabemos que nos comunicamos con otras personas (sin esto el escéptico ni siquiera puede hacer inteligible su duda). Además, es un requisito de la comunicación que se asuma que el hablante sabe lo que quiere decir con sus proferencias. Así, si el hablante sabe que presenta como verdadera la oración que profiere y sabe lo que quiere decir con ella, entonces sabe lo que cree (i.e., tiene conocimiento sobre sus estados mentales). De modo que hay una presunción, esencial para la posibilidad de interpretación $-\mathrm{y}$, por ende, para la comunicación misma-, de que el hablante sabe lo que cree cuando afirma que cree algo.

El argumento de McKinsey rechaza exitosamente a $\left(\mathrm{AC}_{2}\right)$ y muy probablemente a $\left(\mathrm{AC}_{1}\right),{ }^{10}$ pero no contiene ningún elemento que sugiera que $\left(\mathrm{AC}_{3}\right)$ es también incompatible con $(\mathrm{E})$.

\footnotetext{
${ }^{9}$ Se ha insinuado también que una buena forma de presentar la propuesta de Davidson a este respecto es como si se tratase de un argumento trascendental; e.g., Maker 1991, Hacker 1997 y Cutrofello 1999. Sin embargo, el mismo Davidson (1999) considera que ésta no es una presentación idónea y que en principio el argumento no fue planteado de esa manera. He preferido exponerlo como una inferencia a la mejor explicación. Sigo esta forma de presentar la tesis davidsoniana por sugerencia de Jorge R. Tagle Marroquín. Para una discusión crítica en torno a los argumentos trascendentales ver Chisholm 1978.

${ }^{10}$ Es oportuno indicar que McKinsey defiende una versión debilitada de (AC) en 2002a; 46 ss.; y 2007. Sin embargo, se trata de una versión exageradamente restringida, que se aplica sólo a estados mentales en sentido estrecho (los cuales no han sido objeto de discusión en el presente documento). Ver n. 1 .
} 


\section{Conclusiones}

Si la propuesta de McKinsey es compatible con una versión de (AC) respecto a pensamientos amplios, ¿por qué no hace explícita esta compatibilidad? Como lo veo, existen al menos dos alternativas:

1) La versión de (AC) compatible con (E) no puede reconstruirse desde la perspectiva de McKinsey.

2) McKinsey no está interesado en desarrollar esta versión de (AC), aunque puede hacerlo.

Encuentro (2) muy poco plausible, pues una reconstrucción tal abonaría a favor de la reductio: nos daría una imagen refinada de (AC) que descartaría los elementos que entran en contradicción con $(\mathrm{E})$; esto es, rescataría la fuerte intuición que tenemos sobre nuestro privilegio en la autoadscripción de estados mentales sin renunciar a la también fuerte inclinación que conservamos hacia el externalismo sobre el contenido. Me veo, pues, tentado a optar por (1).

No obstante, esto deja una pregunta en el aire: ¿por qué no puede reconstruirse una versión à la Davidson desde la perspectiva de McKinsey? Considero que la respuesta a esto no es sencilla, pues no involucra un simple ajuste local en la postura de McKinsey, sino diferencias radicales en las premisas con las que trabajan ambos enfoques. Enumeraré algunas de estas diferencias, sin entrar en detalles.

a) Semánticas: siguiendo el ejemplo de Kripke, McKinsey considera que para la comprensión adecuada de una enunciado deben comprenderse: i) las condiciones (extensionalmente correctas) bajo las cuales de hecho es verdadero, así como ii) las condiciones en las que describiría correctamente un curso contrafáctico de sucesos (ver Kripke 1972; 12). Por contraparte, Davidson no parece suponer que para la comprensión adecuada de un enunciado sea necesario el segundo requisito. Parte importante y no trivial de ambos argumentos reside en consideraciones semánticas, y en este ámbito no es una cuestión resuelta cuál es la mejor elección en el tratamiento de las actitudes proposicionales. ${ }^{11}$

${ }^{11}$ Hay que precisar que estos tratamientos no son las únicas opciones disponibles en el ámbito filosófico actual, ni se encuentran exentas de costos y compromisos suplementarios. Hay 
b) Debido a que una exposición adecuada de las diferencias epistemológicas exigiría un tratamiento considerablemente amplio y detallado, las dejaré a un lado de momento.

c) Ontológicas: mientras McKinsey trabaja con el modelo casi ecuménicamente aceptado de la ontología de propiedades, Davidson, haciendo eco de Quine, considera que este modelo es sumamente problemático, pues no se han definido claramente los criterios para individuar propiedades (esto quizá sea más nítido a la luz de la discusión sobre propiedades relacionales). (Ver n. 4).

d) Metodológicas: McKinsey, como muchos partidarios de la semántica de la referencia directa, considera que una forma interesante de plantear los problemas filosóficos es, de entrada, fijar una ontología básica y - al menos así lo consideran muchos de sus partidarios - poco problemática: la ontología de propiedades. Partiendo de esto habría que hacer consideraciones semánticas y epistémicas, de modo que estas últimas trabajen a la par y no entren en contradicción con aquélla, dándonos finalmente una imagen clara de la realidad. Davidson, por su parte, asume que podemos clarificar la ontología por medio de una teoría semántica haciendo consideraciones epistemológicas, pero no es necesario transferir lo que resulte de esta teoría y estas consideraciones al plano ontológico (no se debe confundir relevancia explicativa con eficacia causal). De este modo, Davidson rescata las observaciones de Quine sobre la inescrutabilidad de la referencia. ${ }^{12}$

otras concepciones igualmente viables (aunque tampoco libres de crítica): tal es el caso de una semántica de las condiciones de verdad à la Frege y de la semántica del rol conceptual. De igual manera, la naciente semántica de dos dimensiones seguro tendrá algo que decir sobre este punto (muy probablemente en favor de la compatibilidad, si se considera la orientación que sus partidarios han ido delineando).

${ }^{12}$ Creo que se podría ilustrar parcialmente esta diferencia metodológica por medio de una analogía con las teorías clásica y bayesiana de la probabilidad. De manera similar a como he considerado la estrategia de la referencia directa con respecto a la ontología, la teoría clásica de la probabilidad comienza por asignar valores fijos a las diversas probabilidades (e. g., con respecto al lanzamiento de una moneda, asigna $1 / 2$ a cada uno de los posibles resultados de lanzar la moneda [cara o cruz]). Luego hace predicciones y genera explicaciones en virtud de este modelo y trabaja bajo el supuesto de que, entre más se aproxime el número de lanzamientos a $\infty$, tanto más se irá regularizando la distribución de los valores para cada una de las variables, aproximándose a la asignación original. Sin embargo, si en un número importante de casos la diferencia con la asignación original es significativa (digamos que de 1`000 
Partiendo de las premisas de Davidson es posible reconstruir tanto (E) como (AC3) sin que se llegue a la incompatibilidad, esto es, la versión davidsoniana permite conservar el externalismo sin renunciar a la autoridad de la primera persona. Aun más, la versión de Davidson no es incompatible con (AC1) y (AC2), podría defender una de estas formulaciones del autoconocimiento - hasta cierto punto-, pero no lo necesita. Estas consideraciones no son suficientes para rechazar la versión McKinsey, pero sí - al menos en los términos aquí expuestos - para preferir la de Davidson.

En última instancia creo que Davidson sería más radical y más benevolente que McKinsey en lo que respecta al problema de la compatibilidad entre (AC) y (E). Más radical, pues se negaría a discutir el problema de la autoridad de la primera persona en términos ontológicos y epistemológicos - como sí lo hace McKinsey-, es decir, quizá no le concedería ninguna realidad en estos términos (aunque tampoco se la negaría - como sí lo hace McKinsey). Pero más benevolente, en la medida en que reconoce que nuestras intuiciones acerca del conocimiento privilegiado de los propios estados mentales pueden tener valor metodológico para esclarecer ciertos asuntos concernientes a la semántica y la comprensión del lenguaje de nuestros semejantes. Sin embargo, esto requiere una mayor elaboración.

\footnotetext{
lanzamientos, 125 caen cara y 875, cruz), heurísticamente la teoría clásica de la probabilidad asume una desviación (que la moneda está deforme o mal equilibrada) y reasigna valores fijos en función del último resultado en consideración (1/8 para cara y 7/8 para cruz). Por contraparte, análogamente a la metodología de Davidson en lo relativo a la ontología, la teoría bayesiana inicia con una asignación arbitraria de valores a las diversas posibilidades (aunque por lo general comienza con una distribución equitativa), y ajusta estos valores en función de las nuevas experiencias, luego hace predicciones y genera explicaciones en virtud de este modelo y trabaja bajo el supuesto de que entre más se aproxime el número de lanzamientos a $\infty$, tanto más se irá regularizando la distribución de los valores para cada una de las variables, aproximándose a la distribución real. De modo que la teoría bayesiana no necesita comprometerse con una distribución fija de probabilidades, ni hacer suposiciones sobre las causas de una desviación significativa con respecto al estimado inicial. Podría hacerlo, pero no lo requiere ni esto amplía su capacidad explicativa. Agradezco a Ramón Jiménez Lara su instructiva y paciente explicación de las diferencias entre estas dos teorías de la probabilidad, aunque temo no hacerle justicia en los detalles.
} 


\section{BIBLIOGRAFÍA}

Burge, T. (1988). Individualism and Self-Knowledge. The Journal of Philosophy 85 (11), 649-63. Reimpreso en 1998. Externalism and Self-Knowledge.

Burge, T. (1979). Individualism and the Mental. Midwest Studies in Philosophy 4, 73-122. Reimpreso en 1998. Externalism ans SelfKnowledge. [Versión castellana: (1996). El individualismo y lo mental, S. Saab (trad). En M. Valdés (comp), Pensamiento y lenguaje: problemas en la atribución de actitudes proposicionales (pp. 311-382). México: UNAM-IIF].

Chisholm, R. M. (1978). What is a Trascendental Argument? Neue Hefte für Philosophie 19, 19-22. [Versión castellana: (1999). ¿Qué es un argumento trascendental? En I. C. Villoro (comp), Argumentos trascendentales (pp. 85-90). México: UNAM-IIF].

Cutrofello, A. (1999). On the Transcendental Pretensions of the Principle of Charity. En L. E. Hahn (ed), The Philosophy of Donald Davidson. Illinois: Open Court Publishing Company.

Davidson, D. (1999). Reply to Andrew Cutrofello. En L. E. Hahn (ed), The Philosophy of Donald Davidson. Illinois: Open Court Publishing Company.

Davidson, D. (1987). Knowing One's Own Mind. Proceedings and Addresses of the American Philosophical Association 60 (3). Reimpreso en 1998. Externalism and Self-Knowledge. [Versión castellana: Davidson, D. (1992). Mente, mundo y acción. Barcelona: Paidós].

Davidson, D. (1984). First Person Authority. Dialectica 38 (2-3), 101-12. Reimpreso en Davidson, D. (2004). Subjective, Intersubjective, Objective (pp. 3-14). New York: Clarendon Press. [Versión castellana: (1989). La autoridad de la primera persona. En E. Villanueva (comp.), Cuarto Simposio Internacional de Filosofía. Vol. II. México: UNAM-IIF].

Gertler, B. (2003). Self-Knowledge. Stanford Encyclopedia of Philosophy. [http://plato.stanford.edu/entries/selfknowledge/\#5J].

Hacker, P. M. S. (1997). Davidson on First-Person Authority. The Philosophical Quarterly 47 (188), 265-304.

Kripke, S. A. (1972). Naming and Necessity. En D. Davidson \& G. Harman (eds), Semantics of Natural Language. Boston: Reidel. Reim- 
preso en: Kripke, S. A. (1980). Naming and Necessity. Massachusetts: Harvard University Press. [Versión castellana: Kripke, S. (2005). El nombrar y la necesidad. M. M. Valdés (trad). México: UNAM-IIF].

Lau, J. (2003). Externalism About Mental Content. Stanford Encyclopedia of Philosophy. [Online resource: http://plato.stanford.edu/ entries/content-exrernalism/\#6]

Ludlow \& Martin (eds). (1998). Externalism and Self-Knowledge. Stanford: CSLI Publications.

Maker, W. (1991). Davidson's Transcendental Arguments. Philosophy and Phenomenological Research 51 (2), 345-60.

McKinsey, M. (2007). Externalism and Privileged Access are Inconsistent. En B. McLaughlin \& J. Cohen (eds), Contemporary Debates in the Philosophy of Mind. Oxford: Blackwell.

McKinsey, M. (2002a). The Semantic Basis of Externalism. En D. Shier, J. Keim Campbell \& M. O'Rourke (eds), Meaning and Truth. Investigations in Philosophical Semantics. Michigan: Seven Bridges Press.

McKinsey, M. (2002b). On Knowing Our Own Minds. The Philosophical Quarterly 52 (206), 107-16.

McKinsey, M. (1995). Anti-Individualism and Privileged Access. Analysis 51, 9-16. Reimpreso en 1998. Externalism and Self-Knowledge.

McLaughlin \& Tye. (1998). The Brown-McKinsey Charge of Inconsistency. En S. McDonald \& C. Wright (eds). Knowing Our Own Minds: Essays in Self-Knowledge. New York: Oxford University Press. Reimpreso en Externalism and Self-Knowledge.

Parent, T. (2007). Infallibilism about Self-Knowledge. Philosophical Studies 133, 411-24.

Putnam, H. (1975). The Meaning of 'Meaning'. En K. Gunderson (ed), Language, Mind and Knowledge. Minnesota: Minnesota University Press. Reimpreso en Putnam, H. (1975). Mind, Language and Reality. En Philosophical Papers. Vol. 2. Cambridge: Cambridge University Press. [Versión castellana: Putnam, H. (1984). El significado de «significado». J. G. Flematti (trad). México: UNAMCrítica]

Wheatherson, B. (2006). Intrinsic vs. extrinsic properties. Stanford Encyclopedia of Philosophy. [http://plato.stanford.edu/entries/intrinsic-extrinsic/] 


\section{RESUMEN}

En décadas recientes se ha considerado que una formulación adecuada del autoconocimiento (AC) debería ser consistente con la tesis del externalismo (E). Michael McKinsey es uno de los personajes que ha enfatizado con mayor ahínco que la conjunción de ambas posturas es inconsistente. En este trabajo defiendo la idea de que las objeciones presentadas por McKinsey no afectan de manera importante la formulación davidsoniana de la autoridad de la primera persona $\left(\mathrm{AC}_{3}\right)$ en conjunción con (E); señalo, además, que si no pudiera hacerse una reconstrucción à la Davidson de (AC3) desde la postura de McKinsey sería en virtud de las diferencias metodológicas, semánticas, epistemológicas y ontológicas entre ambos autores, y no debido a que esta reconstrucción en sí misma sea incompatible con el externalismo.

Palabras clave: Davidson; McKinsey; externalismo; autoridad de la primera persona; autoconocimiento.

\section{ABSTRACT}

In recent decades it has been considered that a sound formulation of self-knowledge should be consistent with the thesis of externalism. Michael McKinsey has strongly emphasized that the conjunction of both views is inconsistent. In this paper I argue that the objections presented by McKinsey do not importantly affect the Davidsonian account of first-person authority in conjunction with externalism; besides, I point out that if there couldn't be a à la Davidson reconstruction of first-person authority from McKinsey's point of view, it would be in virtue of methodological, semantic, epistemic and ontological differences between both thinkers, and not because this reconstruction is in itself incompatible with externalism.

Keywords: Davidson; McKinsey; externalism; first-person authority, self-knowledge. 
